\title{
Correction to: Radiofrequency identification tag system improves the efficiency of closed vitrification for cryopreservation and thawing of bovine ovarian tissues
}

T. Sato ${ }^{1} \cdot$ Yodo Sugishita $^{1,4} \cdot$ Y. Suzuki $^{1} \cdot$ M. Kashiwagi ${ }^{1} \cdot$ S. Furuyama ${ }^{1} \cdot$ S. Nishimura ${ }^{1} \cdot$ A. Uekawa ${ }^{1} \cdot$ T. Koizumi $^{1}$ $^{2}$ M. Awaji ${ }^{3} \cdot$ T. Sawa $^{2} \cdot$ A. Tozawa ${ }^{1} \cdot$ V. Komatsu ${ }^{2} \cdot$ Nao Suzuki $^{1}$ (D)

Published online: 30 December 2020

(C) Springer Science+Business Media, LLC, part of Springer Nature 2020

Correction to: Journal of Assisted Reproduction and Genetics (2019) 36:2251-2257

https://doi.org/10.1007/s10815-019-01599-3

The original article unfortunately has a missing information. T. Sato and Yodo Sugishita contributed equally to the manuscript.

Publisher's note Springer Nature remains neutral with regard to jurisdictional claims in published maps and institutional affiliations.

The online version of the original article can be found at https://doi.org/ 10.1007/s10815-019-01599-3

Nao Suzuki

nao@marianna-u.ac.jp

1 Department of Obstetrics and Gynecology, St. Marianna University School of Medicine, 2-16-1 Sugao, Miyamae-ku, Kawasaki City, Kanagawa 216-8511, Japan

2 KRD Corporation, City, Yamato, Kanagawa 242-0007, Japan

3 Its Corporation, Kawasaki City, Kanagawa 211-0041, Japan

4 Department of Frontier Medicine Institute of Medical Science, St. Marianna University, School of Medicine, Kawasaki

City, Kanagawa 216-8511, Japan 